\title{
Die Wiedertäuferbewegung1
}

\author{
von Hermann EhMer
}

\section{Vorbemerkung}

Bei diesem Thema ist eine Vorbemerkung zur Begrifflichkeit notwendig. Seit einiger Zeit ist es üblich, nicht mehr von den Wiedertäufern, sondern von den Täufern zu reden. Dieser Wandel ist 1948 auf Wunsch des Mennonitischen Geschichtsvereins zustande gekommen, der nach dem Zweiten Weltkrieg half, die Arbeit des Vereins für Reformationsgeschichte auf diesem Gebiet wieder in Gang zu bringen. Man wies darauf hin, dass nicht die Wiedertaufe, sondern die Erwachsenentaufe das Kennzeichen der Täuferbewegung ist. ${ }^{2}$ Diese verstehen sich als Taufgesinnte, wie die Eigenbezeichnung lautet. Dieser terminologische Wechsel war jedoch im Englischen nicht möglich, wo man nach wie vor von den Anabaptists redet, da Baptists ja eine in der Gegenwart bestehende Kirche meint. ${ }^{3}$

Die Täufer zusammen mit Spiritualisten und Chiliasten in einen ,linken Flügel der Reformation“4 einzuordnen, hat sich nicht bewährt, weil sich mit diesem Begriff stets politische Vorstellungen verbinden. Der Begriff Wiedertäufer oder Anabaptisten hingegen ist ein Quellenbegriff des 16. Jahrhunderts. Er meint diejenigen Gruppen, die die Erwachsenentaufe üben an Menschen, die bereits als Säuglinge getauft worden sind. Der Begriff der Wiedertäufer rückt freilich die Taufe in den Mittelpunkt, obgleich diese nicht die zentrale Frage darstellt, wie noch gezeigt werden soll.

\section{Entstehung des Täufertums}

Die täuferischen Gruppen der Reformationszeit stellten eine kleine Minderheit dar, die freilich die Einheit von Kirche und Gesellschaft in Frage stellten und

1 Vortrag bei der Tagung „Aspekte der Reformation, Johannes Brenz, Primus Truber und der deutsche Südwesten, Schwäbisch Hall, 17./18. März 2017“. Für den Druck wurde der Text erweitert und mit Nachweisungen versehen.

2 Vgl. das Geleitwort zu Manfred Krebs: Quellen zur Geschichte der Täufer, Bd. 4: Baden und Pfalz (Quellen und Forschungen zur Reformationsgeschichte 22 = Quellen zur Geschichte der Täufer 4). Gütersloh 1951, S. V-VII.

3 Vgl. James M. Stayer und Hans-Jürgen Goertz, Art. Täufer/Täuferische Gemeinschaften. In: Theologische Realenzyklopädie 32 (2001), S. 597-623, hier 1.1 Begriff.

4 Heinold Fast: Der linke Flügel der Reformation. Glaubenszeugnisse der Täufer, Spiritualisten und Antitrinitarier (Klassiker des Protestantismus 4). Bremen 1962. 
deshalb verfolgt wurden. ${ }^{5}$ Die Täuferbewegung ist höchst vielfältig. ${ }^{6}$ Die Ursprünge liegen in der ersten Täufergemeinde, die 1524/25 in Zürich entstand. Die Zürcher Reformation durch Ulrich Zwingli erfolgte im Bunde mit dem Rat, der Vertretung der politischen Führungsschicht. Es handelte sich somit um eine obrigkeitliche Reformation. Den politisch Handelnden standen gebildete Laien und Geistliche gegenüber, die gegen den Rat misstrauisch waren. In dieser Gruppe sind Konrad Grebel und Felix Mantz namhaft zu machen, die in Verbindung mit den Wittenbergern Karlstadt und Müntzer standen. Ihnen ging die Zwinglische Reformation nicht weit genug. Sie forderten die Abschaffung von Pfründen und Zehnten, eine autonome Ortsgemeinde und freie Pfarrerwahl. Die letztere Forderung stand dann auch 1525 an der Spitze der Zwölf Artikel der Bauernschaft in Schwaben. Die Erfüllung dieser Forderungen hätte freilich in Zürich die Herrschaft der Stadt über das Landgebiet gefährdet.

Die Täufer forderten den Gehorsam gegen das Evangelium der Bergpredigt. Sie suchten in der Nachfolge die heilige Gemeinde in der Absonderung von der Welt zu verwirklichen. Grundlage der Taufe sollte der Glauben sein, die Entscheidung für die Nachfolge Christi, eine Bekehrung. Deshalb wurde die Erwachsenentaufe geübt und die Kindertaufe abgelehnt.

\section{Verfolgung und Ausbreitung}

Die Züricher Gruppe wurde verfolgt, Todesstrafen wurden vollstreckt, das erste Opfer war Felix Mantz. Die Flucht vieler Züricher Täufer förderte die weitere Ausbreitung der täuferischen Gedanken, vor allem nach Straßburg und Augsburg. Zum zweiten Zentrum des Täufertums wurde Waldshut unter Balthasar Hubmaier (ca. 1485-1528), der seine Gemeinde zu einer täuferischen umbildete. Waldshut wurde aber 1525 im Zusammenhang mit dem Bauernkrieg durch den habsburgischen Stadtherrn belagert und eingenommen. Hubmaier konnte fliehen und begab sich nach Mähren, das für die täuferische Geschichte von großer Bedeutung werden sollte.

Wilhelm Reublin von Rottenburg, Priester in Basel, war ein besonders eifriger Täufer. 1526 begab er sich nach Horb, wo er die Erwachsenentaufe übte. Besonders bedeutsam für die Organisation der täuferischen Gemeinde wurde dort Mi-

5 Claus-Peter Clasen: Die Wiedertäufer im Herzogtum Württemberg und in benachbarten Herrschaften. Ausbreitung, Geisteswelt und Soziologie (VKfgL B 32). Stuttgart 1966, S. 27, zählt im Herzogtum Württemberg, in der Grafschaft Hohenlohe und den von diesen Territorien eingeschlossenen ritterschaftlichen Dörfern zwischen 1529 und 1534 insgesamt 135 Täufer. Obwohl von einer gewissen Dunkelziffer auszugehen ist, bleibt die Täuferbewegung immer noch im Promillebereich der Gesamtbevölkerung.

6 Zur Entstehung des Täufertums im Rahmen der Reformationsgeschichte vgl. jetzt: Thomas Kaufmann: Erlöste und Verdammte. Eine Geschichte der Reformation. München ${ }^{2} 2017$, S. 172-176. Zur geschichtlichen Entwicklung der Täuferbewegung im Herzogtum Württemberg und den benachbarten Herrschaften vgl. Clasen (wie Anm. 5), S. 1-51. 
chael Sattler, auf den die Schleitheimer Artikel, eine täuferische Gemeindeordnung, zurückgehen. Die Schleitheimer Artikel wurden 1527 von einer Täuferversammlung in Schleitheim am Randen (bei Schaffhausen) beschlossen. ${ }^{7}$ Gefordert wird hier die Absonderung von der Welt als bewusster Akt, der die Erwachsenentaufe voraussetzt. Der Bann, also die Ausweisung aus der Gemeinschaft, ist das Instrument der Gemeindezucht. Das Brotbrechen wird in gut zwinglischer Tradition als Gedächtnismahl verstanden. Das Amt der Gemeindeleitung wird eingerichtet. Es wird verneint, dass es eine christliche Obrigkeit geben könne. Entsprechend den evangelischen Räten leisten die Täufer keinen Eid. Die Schleitheimer Artikel bilden die Lehrgrundlage der so genannten Schweizer Brüder, die im Wesentlichen die oberdeutschen Täufer bildeten. Die zweite oberdeutsche Gruppe bildeten die Hutterer, die nach dem Tiroler Jakob Huter $(† 1536)$ genannt werden und sich vor allem durch ihre in Mähren gegründeten Gemeinschaftssiedlungen von anderen Täufern unterscheiden.

Die Horber und Rottenburger Täufer wurden noch 1527 gefangengenommen und ihnen in Rottenburg der Prozess gemacht. Sattler wurde verbrannt, seine Frau im Neckar ertränkt, vier weitere Täufer wurden geköpft. Mit diesem rigorosen Vorgehen wurden die kaiserlichen Dekrete gegen die Täufer, die 1528/29 erschienen, gewissermaßen vorweggenommen.

Das Mandat des kaiserlichen Reichsregiments vom 4. Januar 1528, ${ }^{8}$ das im Namen Kaiser Karls V. ausging, macht den verhängnisvollen Schluss von der Wiedertaufe zum Aufruhr, nämlich das si in schein solcher irer irrigen sect des widertaufs all ober- und erberkeit, auch gemain ruwig wesen, ordnungen und policei abzutun, underzutrucken und zu verwüsten gesonnen seien. Damit war die Richtung gewiesen für die weiteren kaiserlichen Verlautbarungen, wie dem auf dem Reichstag zu Speyer am 23. April 1529 erlassenen Mandat, ${ }^{9}$ das dem Reichsabschied vom 22. April $1529^{10}$ einverleibt wurde, das folgendermaßen lautet:

Nachdem auch kurzlich ain neu sect des widertaufs entstanden, so in gemainem rechten verpoten und vor vil hundert jarn verdampt worden ist, welche sect uber ksl. ausgangen mandat je lenger je mer swerlicher einbricht und uberhand nimpt, und dann ir m. solch swer ubel und waz daraus volgen mag zufurkumen und frid und ainigkeit im heiligen reich zuerhalten, ain rechtmeßig constitucion, satzung und ordnung aufgericht und allenthalb im h. reich zuverkunden verschafft also lautend, das alle und jede widertaufer und wider getaufte mannen und weibspersonen verstendigs alters von naturlichem leben zum tot mit

7 Kaufmann (wie Anm. 6), S. 329.

8 Gustav Bossert: Quellen zur Geschichte der Wiedertäufer, I. Band: Herzogtum Württemberg (Quellen und Forschungen zur Reformationsgeschichte 13). Leipzig 1930, Nr. 1, S. 1*f.

9 Ebd., Nr. 3, S. 3*-5*.

10 Deutsche Reichstagsakten. Jüngere Reihe, Bd. 7, 1. Halbband bearb. von Johannes Kühn. Göttingen ${ }^{2}$ 1963, S. 1298-1314, hier S. 1299 f. - Abdruck der einschlägigen Stelle auch bei Bossert (wie Anm. 8), Nr. 2, S. $2 *$ f. 
dem feuer, schwert oder dergleichen nach gelegenhait der personen on vorgeend der geistlichen richter inquisicion gericht und gepracht werden, und sollen derselben fridprecher haubtsacher, landlaufer und die aufrurigen aufwigler des berurten lasters des widertaufs, auch die, so darauf beharren oder zum andern mal umbgefallen, in solchem kains wegs begnadet sonder gegen inen vermug solcher satzung ernstlich mit straf gehandlet werden; welche person aber iren irrsal fur sich selbs oder auf underricht und ermanung unverzuglich bekennten, denselben zuwideruefen auch pueß und straf daruber anzunemen willig sein und umb gnad pitten wurden, daz dieselbigen nach gelegenhait ires stands, wesens, jugend und allerlai umbstand mugen begnadet werden; daz auch ain jeder sein kinder nach ehr, ordnung, herkumen und geprauch in der jugent taufen lassen sol, welche aber daz verachten und nit tun wurden auf mainung, als sol derselbig kindertauf nicht sein, daz dieselben, so darauf zu beharren understuenden, fur ainen widertaufer geacht und obangezaigten ksl. constitucion underworfen sein, und sol kainer derselbigen, so aus obangezaigten ursachen begnad werden, an andere ort relogiert und verwisen, sonder under seiner obrigkait zu bleiben verstrickt und verpunden werden, die dann ain vleißig aufsehen haben sollen, damit si nit widerumb abfallen.

Dergleichen daz kainer des andern undertanen oder verwonten, so des widertaufs halber von irer oberkait gewichen oder ausgetreten, enthalten, underslaifen oder furschueben, sonder alspald dieselbig obrigkait, darunder sich der entwichen enthelt, solch uberfarung innen oder gewar wirdet, sol er gegen demselben so entwichen, laut obberuerter ksl. satzung strenglich handlen und si daruber nit bei sich leiden oder dulden, alles bei pene der acht etc. Daz demnach wir auf kff., ff., prelaten, graven und stände uns ainmuetiglich verglichen, solcher ksl. constitucion, ordnung und satzung in allen oberzelten puncten und artiklen treulich und vleißiglich zugeleben, nach zukumen zun zuvolziehen.

Die Angelegenheit der Täufer erscheint in dieser Weise auch weiterhin in den Abschieden der Reichstage zu Augsburg 1530, Worms 1535, Speyer 1544, Augsburg 1551 und $1566 .{ }^{11}$ Mit der Todesstrafe für die Täufer wurde die spätantike Ketzergesetzgebung aufgegriffen, die unter Kaiser Theodosius I. (379-395), dann besonders in den Auseinandersetzungen in der nordafrikanischen Kirche mit den Donatisten um 400, also zur Zeit des heiligen Augustinus unter dem weströmischen Kaiser Honorius (395-423) in Anwendung gekommen war.

Die kaiserliche Gesetzgebung war auch maßgebend bei dem Vorgehen gegen eine weitere täuferische Gruppe, die in Esslingen, vor allem in den Filialorten entstanden war. Diese Gruppe erwartete für Pfingsten 1528 das Gericht der Frommen über die Gottlosen. Die potentielle Gewaltbereitschaft dieser Gruppe im Gegensatz zu den pazifistischen Schweizer Brüdern, schürte bei den Obrigkeiten die Angst vor einem täuferischen Aufstand. Sechs dieser Täufer wurden

11 Die entsprechenden Abschnitte sind abgedruckt bei Bossert (wie Anm. 8), Nr. 4-8, S. 5*-10*. 
1529/30 in Esslingen hingerichtet. Andere wurden ausgewiesen und wandten sich nach Reutlingen, wo sie jedoch von Alber bekehrt wurden. ${ }^{12}$ Einzelne wichen nach Heilbronn aus. Sowohl für die Katastrophenpropheten wie für die harte Reaktion der Obrigkeit ist zu bedenken, dass 1529 die Türken vor Wien standen.

Ein Zentrum des Täufertums wurde auch die Reichsstadt Augsburg, wohin das Täufertum durch Balthasar Hubmaier gebracht worden war. Von Augsburg aus bildete sich eine täuferische Gruppe in Schwäbisch Gmünd. Auch in Schwäbisch Gmünd fand 1529 ein Täufergericht statt, wobei sieben Personen hingerichtet wurden $^{13}$. Einzelne Täufer gab es auch im Herzogtum Württemberg, in Stuttgart und Umgebung, im Remstal und in der Göppinger Gegend. Diese wurden während der österreichischen Zeit (bis 1534) ebenfalls verfolgt, wobei es auch zu Hinrichtungen kam.

$\mathrm{Zu}$ erwähnen ist hier noch Augustin Bader, der jedoch einen Einzelfall darstellt. Er gehörte ursprünglich zu den Augsburger Täufern, machte dann aber während eines Aufenthalts in Lautern bei Blaubeuren eine eigenständige Weiterentwicklung durch. ${ }^{14}$ Angeregt von Hans Hut und Wormser Juden sah er seinen neugeborenen Sohn als den Messias an, er ließ für ihn königliche Insignien anfertigen und sah den revolutionären Umsturz für 1530 voraus. Bader wurde gefangengenommen und 1530 auf dem Marktplatz in Stuttgart mit dem für seinen messianischen Sohn angefertigten Königsschwert hingerichtet. Bader bot den Anlass dafür, dass in Artikel 17 des Augsburger Bekenntnisses von 1530 der Chiliasmus verworfen wurde: Item, werden hie verworfen auch etlich judisch Lehren, die sich auch itzund eräugen, daß vor der Auferstehung der Toten eitel Heilige, Fromme ein weltliches Reich haben und alle Gottlosen vertilgen werden. ${ }^{15}$

\section{Johannes Brenz und die Täufer}

Einen anderen Umgang mit den Täufern begründete Johannes Brenz in Schwäbisch Hall. ${ }^{16}$ Er erstellte 1528 für Nürnberg, genauer für den dortigen Ratsschreiber Lazarus Spengler ein Gutachten Ob ein weltliche Oberkeit mit Götlichem und billichen rechten möge die Widerteuffer durch fewr oder schwert vom leben zu dem Tode richten lassen. Die Frage war, ob Ketzer und Wiedertäufer von der

12 Vgl. dazu James M. Stayer: Eine fanatische Täuferbewegung in Esslingen und Reutlingen? In: BWKG 68/69 (1968/1969), S. 53-59.

13 Hermann Ehmer: Das Gmünder Täufergericht 1529. In: Gmünder Studien 1 (1976), S. 131-161. 14 Gustav Bossert: Augustin Bader von Augsburg, der Prophet und König, und seine Genossen, nach dem Prozeßakten von 1530. In: Archiv für Reformationsgeschichte 10 (1912/13), S. 117-165, 209-241, 297-349; 11 (1914), S. 19-64, 103-133, 176-199.

15 Die Bekenntnisschriften der evangelisch-lutherischen Kirche. ${ }^{5}$ Göttingen 1963, S. 72.

16 Zum Folgenden, soweit nichts anderes angemerkt: Gustav Bossert: Johann Brenz, ,der Reformator Württembergs“" und seine Toleranzideen. In: BWKG 15 (1911), S. 150-161; 16 (1912), S. 2547. 
weltlichen Obrigkeit bestraft werden können oder müssen. Nach der Zwei-Reiche-Lehre hat das weltliche Regiment weltliche Vergehen zu bestrafen. Geistliche Sünden wie Ketzerei können nur durch die Predigt bekämpft werden, allenfalls kann man den Bann, den Ausschluss aus der christlichen Gemeinde, über eine solche Person verhängen. Einschlägige Bestimmungen des Alten Testaments, wie Deut. 13, wo von der Todesstrafe der falschen Propheten und der Verführer zum Götzendienst die Rede ist, sind im Neuen Testament geistlich zu verstehen. Das heißt, dass Irrlehre nur mit geistlichen Mitteln, wie der Predigt bekämpft werden kann. Eine Ausnahme ist zu machen, wenn ein Aufruhr geplant wird. Nicht als Aufruhr ist anzusehen, wenn das Gemeineigentum angestrebt wird, weil dies den eigenen Entschluss der Beteiligten voraussetzt. Wird der Eid verweigert oder das obrigkeitliche Amt nicht anerkannt, handelt es sich um Irrtümer, denen man mit Belehrung begegnen muss. Die Wiedertaufe als solche ist keinesfalls der Todesstrafe würdig.

In Nürnberg befanden sich keine Aufrührer unter den Täufern, deshalb war die Entscheidung schwierig. Immerhin stand Brenz mit seiner Auffassung nicht allein. Auch Luther hatte sich 1528 gegen die Todesstrafe ausgesprochen, wobei auch der Aufruhr die Ausnahme bilden sollte. Melanchthon hingegen schrieb den täuferischen Lehren bereits Aufruhrcharakter zu, sodass in seiner Sicht die Todesstrafe gegen Täufer möglich schien.

Ein weiteres Gutachten in der Täuferfrage erstellte Brenz 1530: Ob eine Obrigkeit, wann sie falsche Lehre außrottet, darumb uber die Gewissen herrsche, unnd ob von der Obrigkeit die Irrige wider ir Gewissen können zu anderm Glauben gezwungen werden. Die Veranlassung war vermutlich ein Nürnberger Fall, ein hartnäckiger Täufer, der nicht widerrufen wollte, sich vielmehr auf die Heilige Schrift und sein Gewissen berief. Sollte man gegen ihn mit Landesverweisung oder Haft vorgehen? In diesem Gutachten hat Brenz die Gewissensfrage durchaus differenziert behandelt. ${ }^{17}$ Die Obrigkeit herrscht nicht über den Glauben, hat keinen Gewissenszwang auszuüben, sondern soll helfen, dass die Irrenden zum rechten Glauben kommen. Da Gewissen ein Wissen voraussetzt, geht es darum, zu erkennen, was Gottes Willen und Gesetz ist. Irrtum kann deshalb kein rechtes Gewissen schaffen.

Ein weiterer Nürnberger Fall betraf die Frage der Duldung verschiedener Glaubensgemeinschaften. Nach Brenz sollte es nur ordentlich berufene Prediger und somit keine heimliche Predigt geben. Da Verschiedenheit Unordnung schafft, hat die Obrigkeit für Ordnung zu sorgen. Nach wie vor hat Brenz die Lebensund Leibesstrafe für Täufer abgelehnt, allenfalls könne man auf Ausweisung erkennen.

17 Dazu ebd., S. 25-29; Joseph Lecler SJ: Geschichte der Religionsfreiheit im Zeitalter der Reformation, Bd. 1-2. Stuttgart 1965, hier 1, S. 357-359. 
Die drei genannten Gutachten von Brenz antworten auf konkrete Situationen und können deshalb nicht ohne weiteres verallgemeinert werden. Gemeinsam ist allen drei Äußerungen der Verzicht auf die Todesstrafe, womit sich Brenz gegen die kaiserlichen Mandate aussprach. Brenz hat die Ansichten Luthers breiter entfaltet, sein Gutachten von 1528 ist auch zusammen mit einem solchen von Melanchthon in einem zeitgenössischen Druck erschienen, obwohl es in offenem Widerspruch zur kaiserlichen Gesetzgebung stand. ${ }^{18}$ Damit wirkte Brenz auf die lutherischen Theologen, auch auf Melanchthon. Das weitere Fortwirken dieses Gutachtens war auch durch seine Aufnahme in der von Felix Bidembach 16081611 veranstalteten Sammlung theologischer Gutachten sichergestellt. ${ }^{19}$

Brenz’ Äußerung ging auch ein in das Werk von Sebastian Castellio, De haereticis (Basel 1554), der sich gegen die Verbrennung von Michael Servet (15111553) wandte, die in Genf durch Calvin veranlasst worden war. Brenz wird in Castellios Werk zitiert und hat damit einen Ehrenplatz in der Geschichte der Toleranz erhalten. Es ist dies freilich eine schiefe Sicht. Es geht Brenz nicht um die Existenz andersgläubiger Gruppierungen, sondern um die Frage der Anwendung der Todesstrafe in solchen Fällen, denn nach wie vor hält Brenz die Verantwortung der Obrigkeit für die rechte Lehre und den rechten Gottesdienst aufrecht. ${ }^{20}$ Brenz hat somit nicht eine Wandlung zu geringerer Toleranz gemacht, ${ }^{21}$ vielmehr antwortet er stets auf konkrete Situationen, wobei freilich die Ablehnung der Todesstrafe seine grundlegende Überzeugung bleibt.

Brenz' Vorbild wirkte auch in der Markgrafschaft Brandenburg, wo die Reformation seit 1528 eingeführt wurde, aber zugleich auch Täufer auftauchten. Besonders bedenklich war das Auftreten von Hans Hut (1490-1527), der apokalyptische Vorstellungen von einem unmittelbar bevorstehenden Endgericht hegte. Über diesen Chiliasten urteilten die Theologen durchaus differenziert. Demnach ist der Unglauben an sich nicht strafbar, doch dem täuferischen Lehrer sollte ein Predigtverbot auferlegt werden. Würde ein Unterricht nicht zum Widerruf führen, wäre keinesfalls auf Todesstrafe zu erkennen, allenfalls auf Landesverweisung. Damit ist nicht nur ein Unterschied zur Reichsgesetzgebung festzustellen, sondern auch zu den Wittenbergern, da Melanchthon und Luther eine schärfere Gangart empfahlen.

Im Gebiet der Reichsstadt Schwäbisch Hall waren die Täufer nur von geringer Bedeutung, allenfalls machten sich die württembergischen Durchzügler nach Mähren bemerkbar. ${ }^{22}$

18 Walter Köhler: Bibliographia Brentiana. Bibliographisches Verzeichnis der gedruckten und ungedruckten Schriften des Reformators Johannes Brenz. Berlin 1904, Nr. 29.

19 Felix Bidembach (Hg.): Consiliorum Theologicorum Decas III, IV. Frankfurt 1608, S. 180-200; Köhler (wie Anm. 18), Nr. 730.

20 Bossert (wie Anm. 16) (1912), S. 37; Lecler (wie Anm. 17) 1, S. 347-350.

21 Ebd., S. 349.

22 Clasen (wie Anm. 5), S. $24 \mathrm{f}$. 


\section{Täufer im Herzogtum Württemberg}

Im Herzogtum Württemberg fand unter österreichischer Herrschaft bis 1534 eine strenge Verfolgung statt. König Ferdinand hat schon am 26. Januar 1528 seiner Stuttgarter Regierung eine ausführliche Anweisung gegeben, wie mit den Täufern zu verfahren sei. ${ }^{23}$ Die Regierung gab diesen Befehl am 26. Februar 1528 an die Amtleute weiter. ${ }^{24}$

Nach der Hutterischen Überlieferung wurden bis 1534 im Herzogtum 32 oder 45 Täufer hingerichtet. ${ }^{25}$ Die eingehende Dokumentation des Täufertums im Herzogtum Württemberg ist der umfangreichen Quellensammlung von Gustav Bossert (1841-1925) zu verdanken, dessen posthum erschienenes Werk ähnliche Arbeiten für andere Territorien angeregt hat. Bei der von Bossert gebotenen Fülle des Stoffs ist gleichwohl eine genauere Untersuchung der Quellen notwendig. Die österreichische Verwaltung machte wenig Unterschiede, da alles, was nicht dem hergebrachten Gebrauch entsprach verdächtig war. In manchen Fällen lässt sich deshalb nicht zuverlässig sagen, ob es sich um Anhänger Luthers oder um Täufer handelte.

Seit 1534 wurde in Württemberg bei Täufern nicht mehr auf Todesstrafe erkannt. Die Wiedertäuferordnung vom 18. Juni $1535^{26}$ spricht eine Art Amnestie aus. Nach dem Vorbild etlicher Täufer in den Ämtern Leonberg, Lauffen, Schorndorf und Beilstein wird denjenigen, die ihre Kinder taufen lassen, den Herzog als ihre Obrigkeit anerkennen, zu Predigt und Nachtmahl gehen und keine fremden Prediger herbergen, gegen Ausstellung einer Urfehde Gnade zugesagt.

Ein Mandat, das sich gegen die Wiedertäufer von Münster richtete, wurde am 22. Juni 1535 erlassen. Wohl von 1536 stammt eine „Ordnung der Wiedertäufer" ${ }^{\prime \prime}{ }^{27}$ die Vorschriften für das Verfahren gibt. Die gefangen gesetzten Wiedertäufer sollen, auch „peinlich“, d.h. unter Anwendung der Folter auf eine Reihe von Punkten befragt werden. Es geht hier um Beteiligung am Bauernkrieg, wer die Taufe vorgenommen und wo, und wie viele dabei getauft worden seien. Dann war der Betreffende über seine Meinung von den Sakramenten der Taufe und des Abendmahls zu befragen, desgleichen über die Eidesleistung und ob es eine christliche Obrigkeit gebe. Ferner, ob ein Christ zur Verteidigung des Landes Kriegsdienste leisten könne, ob und inwieweit er der Obrigkeit Gehorsam leisten kann. Dann war zu fragen, was er von Christus halte und ob dieser für unsere Sünden genug getan habe.

Diejenigen Wiedertäufer, die widerrufen, sollten, falls es sich um einfache Leute und nicht um Rädelsführer handle, begnadigt werden. Sie hatten zu verspre-

23 Bossert (wie Anm. 8), Nr. 1, S. 1-4.

24 Ebd., Nr. 3, S. 5-7.

25 Clasen (wie Anm. 5), S. 29, hält diese Zahl für zu hoch gegriffen.

26 Bossert (wie Anm. 8), Nr. 61, S. $41 \mathrm{f}$.

27 Ebd., Nr. 81, S. 57-60. Sammlung der württembergischen Geseze, Bd. 8. Hg. von August Ludwig Reyscher. Tübingen 1834, S. 38-42, setzt diesen Text in das Jahr 1535. 
chen, sich künftig der wiedertäuferischen Lehren zu enthalten und ihre Kinder taufen zu lassen. Zweitens sollten sie den Herzog und seine Amtleute als ihre von Gott verordnete Obrigkeit anerkennen, sich drittens zu Predigt und Nachtmahl halten. Viertens sollten sie keinem fremden Prediger anhängen und solche auch nicht herbergen. Falls sie rückfällig werden, sollten sie an Leib und Leben gestraft werden.

Den Rädelsführern, denen, die ohne Zweifel mit vielen ihrer Sekte zusammengekommen waren, drohte Leibesstrafe. Diese waren über die Namen derjeniger zu befragen, die der wiedertäuferischen Sekte anhängig sind, auch über die Orte, an denen sie zusammenkommen. Es war zu fragen, welcher Gruppe der Betreffende angehörte, denen zu Münster oder zu Mähren oder anderen, und was deren Vorhaben sei. Nachdem viele, die nach Mähren gezogen waren, unter dem Schein des Widerrufs zurückgekommen sind und ihre Güter zurückverlangten, war zu ermitteln, ob sie ausgesandt seien, um Unruhen anzustiften. Auch war nach geheimen Erkennungszeichen zu fragen und ob ein Aufstand gegen die Obrigkeit geplant sei, ob es Ort oder Zeit gebe, wo und wann man sich dazu sammeln wollte. Diejenigen, die des Aufruhrs nicht verdächtig sind, aber nicht widerrufen wollten, sollten des Landes verwiesen werden mit der Drohung, dass sie an Leib und Leben gestraft werden, wenn sie wieder im Land erscheinen. Für das Verhör der Wiedertäufer sollten die Amtleute die geschicktesten und gelertesten predicanten beiziehen, die sie aus der heiligen Schrift unterweisen sollten. Die Prädikanten sollten ihren besten Fleiß anwenden, sollich leut us irem irtumb zu erledigen und zu unseren rechten christlichen glauben wieder zu bringen.

Ein am 13. Juli 1538 erlassenes Generalreskript gegen die Wiedertäufer ${ }^{28}$ lässt erkennen, dass die beiden vorausgegangenen Erlasse recht wenig fruchteten, dass die Wiedertäufer im Land weiterhin zusammenkommen, dass es Rückfällige gibt und solche, die des Landes verwiesen waren und vor allem solche, die aus Mähren zurückgekommen waren, anzutreffen sind. Es wird deshalb die Ordnung erneut eingeschärft.

Diese Ordnung behielt ihre Gültigkeit auch nach 1550 unter der Regierung von Herzog Christoph, der eifrig auf die Reinheit der Lehre bedacht war. Brenz war nun der maßgebende Theologe im Land, unter dessen Mitwirkung das Ordnungswerk mit der Großen Kirchenordnung von 1559 geschaffen wurde. Schon in der Visitationsordnung von $1553^{29}$ war angeordnet worden, auf die ,irrigen secten“ zu achten. In einem Mandat vom 25. Juni 1558, das sich gegen Wiedertäufer, Schwenckfelder und dergleichen wandte, wurde das noch weiter ausgeführt. Es war Ergebnis längerer Beratungen gewesen, auch mit anderen protestantischen Obrigkeiten. Eine gemeinsame Ordnung blieb jedoch auf dem Papier. Die Ordnung von 1558 gibt eine Liste von Frageartikeln. Diese betreffen die Kindertaufe, dann die Sakramente allgemein, ob sie eine Zueignung der göttli-

28 Bossert (wie Anm. 8), Nr. 94, S. $67 \mathrm{f}$.

29 Reyscher 8 (wie Anm. 27), S. 100-105, hier S. 102. 
chen Gnade oder lediglich als Zeichen zu verstehen seien und ob das Predigtamt und die Sakramente überhaupt notwendig sind. Ferner geht es um die Frage der Erbsünde und der Gerechtigkeit vor Gott, ob diese durch Erfüllung des Gesetzes, eigene Werke oder Leiden möglich sei. Hierher gehört auch die Frage, ob die durch die Taufe neu Geborenen nicht wieder Gottes Zorn verfallen können.

Ein weiterer Fragenbereich betrifft die Lehre von Gott, ob die Trinität verworfen wird. Hierher gehört auch die Christologie, die Frage nach der Ubiquität, der Allgegenwart des Auferstandenen und der Inkarnation, der Fleischwerdung. Weiterhin geht es um die Wirkung des heiligen Geistes, ob dieser bei der Wassertaufe wirksam ist, ebenso auch beim Predigtamt, ob hier zwischen äußerlichem und innerlichem Wort unterschieden wird. Desgleichen beim Abendmahl, ob hier zwischen äußerlichem und geistlichem Essen ein Unterschied gemacht wird.

Ein weiterer Bereich ist das Amt der Obrigkeit, ob die weltlichen Ämter unchristlich sind. Hierher wird auch die Frage des Gemeineigentums gezogen, desgleichen ob die Bemühung weltlicher Gerichte unchristlich ist. Dazu gehört auch die Frage der Eidesleistung.

Dieser Fragenkatalog mag auf den ersten Blick viel zu akademisch anmuten. Gewiss werden diese Fragen für manchen zu hoch gewesen sein. Doch wird man sicher sagen können, dass diese Frageartikel Ergebnis der Erfahrung mit den Täufern gewesen seien, die durch Bibellektüre und gegenseitige Unterrichtung theologisch und argumentativ so beschlagen waren, dass es für die Befragung zumindest der täuferischen Lehrer in der Tat der besten Theologen bedurfte.

Die Ordnung von 1558 verweist auf das Württembergische Bekenntnis von 1552, das Augsburger Bekenntnis von 1530 und den Religionsfrieden von 1555, der allein die Anhänger des Augsburger Bekenntnisses in den Frieden einschließt. Erinnert wird ferner an die Reichsabschiede, die Leibesstrafen, Landesverweisung und Güterkonfiskation als Strafen vorsehen. Es ergeht daher an die weltlichen Beamten - und nur an diese - der Befehl, auf Anhänger der irrigen Lehren, auch auf solche, die ihnen Unterschlupf bieten, zu achten und diese gegebenenfalls zu verhaften. Diese Ordnung wurde im wesentlichen unverändert in die Große Kirchenordnung von1559 aufgenommen. ${ }^{30}$

\section{Täuferische Fluchtorte}

Bemerkenswert ist, dass offenbar nicht wenige Adlige, ebenfalls im Widerspruch zu den kaiserlichen Mandaten, Täufer in ihrem Herrschaftsgebiet aufnahmen und duldeten. So arbeitete der aus Heilbronn ausgewiesene Täufer Endris Wertz als Keller oder Verwalter 1530 bei Wilhelm von Massenbach, anschließend bei

30 Neuester Druck: Die evangelischen Kirchenordnungen des XVI. Jahrhunderts. Begr. von Emil Sehling. Bd. 16: Baden-Württemberg II: Herzogtum Württemberg. Bearb. von Sabine Arend. Tübingen 2004, S. 381-384. 
den Neipperg in Schwaigern. ${ }^{31}$ Graf Albrecht von Löwenstein hielt in den 1570er Jahren bis zu seinem Tod 1587 auf seinen Besitzungen, auf der Burg Wildeck und auf dem benachbarten Hof Vohenlohe Täufer als Pächter. Der Graf, der sich der Reformation nicht angeschlossen hatte, wurde 1582 immerhin Obervogt zu Weinsberg. Die aus Stuttgart kommenden Anfragen wegen der bei den Kirchenvisitationen festgestellten Täufer beantwortete er hinhaltend, so dass sich hier offenbar ein täuferischer Sammelpunkt entwickeln konnte, der unter den Täufern im Land bekannt war. ${ }^{32} \mathrm{Ob}$ man hier von Toleranz sprechen kann, muss dahingestellt bleiben. Gewiss spielt hier auch die Behauptung des adligen Eigenrechts gegenüber der herzoglichen Verwaltung eine Rolle. So musste man 1588 einfach zur Kenntnis nehmen, dass Eberhard von Weitershausen in Hohenhaslach einen wiedertäuferischen Maier hatte. ${ }^{33}$ Diese Beispiele ließen sich noch leicht vermehren.

Wegen der Verfolgung wanderten später viele der südwestdeutschen Täufer nach Mähren aus, wo ihnen in der Gegend von Nikolsburg von dem dortigen Adel die Möglichkeit geboten wurde, ihre Ansiedlungen auf der Grundlage des Gemeineigentums zu errichten. ${ }^{34}$ Die Verbindung zwischen Württemberg und Mähren war durch die täuferischen Sendboten sichergestellt, die das Leben in Mähren in anziehender Weise zu schildern wussten, so dass die Leute oftmals bei Nacht und Nebel ihre Heimat unter Zurücklassung ihrer Habe verließen. Wie der Schorndorfer Vogt Sixt Weselin am 2. Juli 1558 berichtete, hatten die Brüder Peter und Endris Sturmer von Rudersberg am 30. Juni in der Nacht mit Frauen und Kindern zusammen mit Peter Lemblins Frau unter Hinterlassung ihrer Habe das Dorf in Richtung Mähren verlassen. Die Leute hatten am Tag zuvor noch auf ihren Äckern gearbeitet, gemäht und Mist getragen. Der Vogt war ihnen nachgeeilt, konnte sie aber nicht mehr erreichen. Im Weiler Ebni erfuhr er, dass man sie dort gesehen hatte. Die Bauern hätten sie aber in der Nacht für Kriegsleute gehalten, weil sie die Bettbezüge, in denen sie ihre Habe eingebunden hatten, für Harnische hielten. Der Vogt konnte sie daher nicht mehr erreichen.

Die hinterlassene Habe der Flüchtigen wurde „verpflegt“, also unter eine besondere Verwaltung gestellt. Cyriakus Föll von Reichenbach schwor in Marbach am 14. Februar 1545 der ,wiedertäuferischen Sekte“ ab. ${ }^{35}$ Föll begab sich hierauf nach Mähren, denn noch 1596 wird seine und die Habe des Friedhans von Reichenbach verpflegt. ${ }^{36}$

31 Clasen (wie Anm. 5), S. 118 f. - Die von Gustav Bossert gesammelten Quellenbelege finden sich im GLA Karlsruhe S Verein für Reformationsgeschichte (Täuferakten) Fasz. 6 B1. 64-98.

32 Hermann Ehmer: Graf Albrecht von Löwenstein (1536-1587). Jerusalempilger und Kriegsunternehmer, Diplomat und Beamter. In: ZWLG 72 (2013), S. 153-226, hier S. 210 f.

33 Bossert (wie Anm. 8), Nr. 914, S. 639.

34 Dazu Clasen (wie Anm. 5), S. 52-62.

35 Bossert (wie Anm. 8), Nachtrag Nr. 86, S. 1013.

36 Ebd., Nr. 977, S. 693. 
Über die hutterischen Siedlungen in Mähren berichtet der württembergische Theologe Stephan Gerlach (1546-1612), der 1573-1578 Prediger bei der kaiserlichen Gesandtschaft in Konstantinopel war und bei der Rückreise in Wischenau bei Znaim seine wiedertäuferischen Geschwister und Verwandte aus Knittlingen besuchte. ${ }^{37}$ Während die Gerlachsche Schilderung sich hauptsächlich auf die von ihm angetroffenen Personen beschränkt, finden wir eine allgemeine Schilderung des Lebens in den täuferischen Siedlungen in Mähren bei Hans Jakob Christoffel von Grimmelshausen in seinem 1669 erstmals erschienenen Roman Der Abentheurliche Simplicissimus. ${ }^{38}$ Für Grimmelshausen stellen die hutterischen Bruderhöfe eine Idealgesellschaft dar, die er angeblich selber in Augenschein genommen, sich aber wegen ihrer „widerwärtigen ketzerischen Meinung“ nicht angeschlossen hatte. Gleichwohl rühmt er das friedliche Zusammenleben der Wiedertäufer: die Handwerker arbeiten auf ihrem Handwerk, der Schulmeister unterrichtet die Jugend, Männer und Frauen gehen getrennt ihrer zugewiesenen Arbeit nach. Es gibt Räume mit Kindbetterinnen und Säuglingen, die beide von Witwen versorgt werden. Es gibt Spinnerinnen, Wäscherinnen und so weiter. Jede Frau und jeder Mann verrichten die ihnen zugewiesene Arbeit. Es gibt bestimmte Zeiten zum Essen, zum Schlafen, aber nicht zum Müßiggang. Mann und Frau sehen sich zu bestimmten Zeiten in dem ihnen zugewiesenen Schlafgemach, aber alle nannten sich Brüder und Schwestern.

\section{Das Täufertum im Herzogtum Württemberg in der zweiten Hälfte des 16. Jahrhunderts}

Trotz der Auswanderung nach Mähren stellten die Täufer in Württemberg noch ein jahrzehntelanges Dauerproblem dar. Aus der städtischen Bewegung war nämlich eine kaum zu überwachende ländliche Subkultur geworden. Täufer fanden sich im evangelischen Württemberg noch jahrzehntelang, vor allem im Remstal und im Schwäbischen Wald. Durch den anderen Umgang mit den Täufern, insbesondere durch den Verzicht auf die Todesstrafe war die Überzeugungskraft der Theologen besonders gefordert.

In Mähren war nicht nur ein Fluchtort entstanden, vielmehr stellte der rege Austausch zwischen den Täufern in Mähren und denen in der Heimat die Obrigkeit und die Theologen vor neue Probleme. Hartnäckige und Wiederholungstäter wurden eingekerkert. Insgesamt sind sechs Personen namentlich bekannt, gegen die in dieser Weise verfahren wurde. Die ungenannte Täuferin, die nach dem Bericht des Vogts von Hohenwittlingen 1555 in ihrer Stube an die Kette gelegt ${ }^{39}$

37 Ebd., Nachtrag Nr. 133, S. 1105-1108.

38 Grimmelshausen, Simplicissimus, 5. Buch, 19. Kapitel.

39 Das Anketten im eigenen Haus wurde gelegentlich verfügt; vgl. z. B. Bossert (wie Anm. 8), Nr. 413, S. 443 . 
wurde, war möglicherweise Barbara Löffler von Stuttgart ${ }^{40}$ die jahrzehntelang die Behörden beschäftigte. Schon 1530 war sie - noch unter der Regierung von König Ferdinand - wegen Verachtung von Taufe, Messe und Beichte in Haft gekommen und musste Urfehde schwören, in der sie versprach, sich künftig an die kirchliche Ordnung zu halten. Zugleich hatte sie eine Kaution von $200 \mathrm{fl} \mathrm{zu}$ hinterlegen. ${ }^{41}$ Aber auch an der Reformation und ihren Vertretern übte sie Fundamentalkritik. Im Gespräch mit Ambrosius Blarer kritisierte sie dessen Kollegen Erhard Schnepf, in dessen Haus sie kein gottseliges Leben erkennen könne. Vor allem nahm sie auch Anstoß daran, dass Schnepfs Frau - immerhin Tochter eines Bürgermeisters der Reichsstadt Wimpfen - samtene Goller und goldene Ringe an den Fingern trage, was Schnepf als Apostel übel anstehe..$^{42}$ Nach ihrer vermutlichen Inhaftierung auf Hohenwittlingen scheint sie alsbald wieder in Freiheit gekommen zu sein, denn bei der Visitation 1563 fiel sie abermals als Wiedertäuferin auf, die Bücher von Täufern und Schwenckfeld besaß, aber sich bereit erklärte, diese auszuliefern. Außerdem hatte sie gegen den Willen ihres Ehemanns wandernde Täufer beherbergt. ${ }^{43}$ Wiederum stellte sie eine Urfehde aus, in der sie versprach, wöchentlich die Predigten zu besuchen. ${ }^{44}$

Ein zweiter Fall ist der des Paul Glock von Rommelshausen, der seit 1550 aktenkundig wird. ${ }^{45}$ Er wurde 1563 eines Verhörs durch Beamte und Theologen unter$\operatorname{zogen}^{46}$, über das er selbst berichtete. Es ist dies eines der vielen Selbstzeugnisse von Glock, zu denen auch seine Briefe aus dem Zeitraum von 1563-1576 gehören. ${ }^{47}$ Glock befand sich seit 1558 in lebenslänglicher Haft, die fast 20 Jahre dauerte, auf Hohenwittlingen. Die durchweg umfangreichen Briefe, die den Charakter von Erbauungsschriften haben, zeigen, dass Glock hinreichend Muße und die Möglichkeit zum Schreiben, aber auch zum Empfang von Briefen aus Mähren gehabt haben muss. Er erwarb sich auf Hohenwittlingen eine Vertrauensstellung, wurde zu Botengängen über Land geschickt, die er auch zu Besuchen unterwegs nutzen konnte. Durch Heimarbeit als Bortenwirker konnte er etwas Geld verdienen. ${ }^{48}$ Auf Hohenwittlingen waren auch Schweizer Brüder inhaftiert, eine andere täuferische Richtung als die Hutterer, zu denen Glock zählte. Hin und wieder ergab sich Gelegenheit zu theologischen Disputen. Einer der

40 Ebd., Nachtrag Nr. 102, S. 1020.

41 Ebd., Nr. 14, S. 13-16.

42 Ebd., Nachtrag Nr. 71, S. 996 f.

43 Ebd., Nachtrag Nr. 112, S. 1048 f.

44 Ebd., Nr. 232, S. 230 f.

45 Ebd., Nr. 149, S. 123.

46 Ebd., Nachtrag Nr. 113, S. 1049-1065.

47 Ebd., Nr. 276 f., S. 334-343; Nr. 282, S. 346f.; Nr. 284-287, S. 348-367; Nr. 401, S. 435; Nr. 474, S. 463 ff.; Nr. 519, S. 477 f.; Nachtrag Nr. 114 f., S. 1065-1081; Nr. 122-126, S. 1086-1102. - Allgemein vgl. dazu auch: Lydia Müller (Hg.): Glaubenszeugnisse oberdeutscher Taufgesinnter (Quellen und Forschungen zur Reformationsgeschichte 20). Leipzig 1938.

48 Vgl. dazu eine Randbemerkung zu einem Gutachten der Regierung 1584; Bossert (wie Anm. 8), Nr. 757, S. 579-581, hier S. 581 Anm. 1. 
Kritikpunkte der Schweizer Brüder war die Gemeinschaftsbildung in Mähren mit der Auflösung des Familienverbands..$^{49} 1576$ half Glock bei einem Brand auf der Burg tatkräftig bei den Löscharbeiten und wurde daraufhin zusammen mit Matthes Binder mit einem Reisegeld nach Mähren entlassen. ${ }^{50}$

$\mathrm{Zu}$ den weiteren Täufern, die längere Zeit inhaftiert waren, gehört Adam Hornickel (Horneck) von Heiningen bei Göppingen, der 1559-1564 vor allem auf Hohenwittlingen gefangen lag. ${ }^{51}$ Matthes Binder war, wie Paul Glock 1576 berichtet, mit ihm schon ein Jahr auf Hohenwittlingen..$^{52}$ Über einen weiteren Gefangenen auf Hohenwittlingen, Michel Hasel, Weber von Rudersberg, der vier Jahre lang, bis zu seinem Tod 1592 dort gefangen war, berichtet die hutterische Überlieferung. ${ }^{53}$ Über ihn ist aus den Akten nichts zu erheben, doch ist anzunehmen, dass er mit weiteren Täufern dieses Namens aus Rudersberg verwandt war. Ganz ähnlich war auch die Maulbronner Gegend ein täuferisches Zentrum. Als ein Vorsteher aus Mähren wird 1574 Simon Kress von Gündelbach namhaft gemacht, dessen Predigten im Brettener und Derdinger Wald die Leute, oft aus „Fürwitz“, besuchten. ${ }^{54}$ Kress befindet sich 1594 im 13. Jahr gefangen auf Hohenwittlingen. Er war zwei- bis dreimal ausgewiesen worden, aber immer wieder gekommen. ${ }^{55}$ Er wird zuletzt 1605 erwähnt. ${ }^{56}$ Von Frau und Sohn, die ihn besuchten, war er bewogen worden, um Entlassung nachzusuchen. Diese wurde ihm auch in Aussicht gestellt, wenn er zwei Bedingungen eingehe, nämlich zu Predigt und Abendmahl zu gehen und sich mit niemanden in eine Disputation einzulassen. Als der Spezialsuperintendent von Urach deswegen nach Hohenwittlingen kam, musste er feststellen, dass Kress nicht mehr bereit war, das Abendmahl zu nehmen, obwohl er ihm noch einen Monat Bedenkzeit gegeben hatte. Er empfahl daher, ihn auch weiter in strenger custodi zu halten.

Seit 1601 wird neben Kress auf Hohenwittlingen auch Hans Tauber (Dauber) von Illingen erwähnt. Er fiel 1581 wegen Lästerungen gegen die Kindertaufe und das Abendmahl auf und kam deswegen nach Maulbronn in Haft, ${ }^{57} 1584$ war er auf Hohenurach ${ }^{58}$ wo er sechs Jahre war. Auf Bitten der Frau wurde er freigelassen und ging 1588 daheim wieder zur Predigt. Obwohl er sich des Abendmahls enthielt, wollte man mit ihm Geduld tragen. ${ }^{59}$ Diese ging aber alsbald zu Ende, denn Dauber kam nach Hohenwittlingen in Haft, wo er 1594 schon fünf

49 Ebd., Nr. 359, S. 412. Vgl. dazu Claus-Peter Clasen: Anabaptists. A Social History, 1525-1618. Switzerland, Austria, Moravia, South and Central Germany. Ithaca and London 1972, S. $40 \mathrm{f}$.

50 Clasen (wie Anm. 5), S. 43, Anm. 202.

51 Bossert (wie Anm. 8), Nr. 263, S. $254 \mathrm{f}$.

52 Ebd., Nr. 474, S. 463.

53 Ebd., Nr. 952, S. 668.

54 Ebd., Nr. 367, S. 417.

55 Ebd., Nr. 961, S. 675.

56 Ebd., Nr. 1018, S. 760 ff.

57 Ebd., Nr. 680, S. 534.

58 Ebd., Nr. 771, S. 587.

59 Ebd., Nr. 915, S. 639. 
Jahre war. ${ }^{60}$ Seit 1601 wird von Dauber und Kress auf Hohenwittlingen berichtet, dass die beiden von Visionen reden und nach Papier verlangen, um diese aufzeichnen zu können. Außerdem behaupten sie, dass Gottes Werk durch sie ausgerichtet werde. Von Dauber sind aus dem Jahre 1596 zwei Briefe an den Pfarrer von Wittlingen erhalten, in denen es um die von seiner Frau beantragte Ehescheidung ging. ${ }^{61}$ Dauber wird zuletzt 1617 auf Hohenwittlingen erwähnt. ${ }^{62}$ Im Ergebnis wird man sagen können, dass die gefangenen Täufer, verglichen mit vielen anderen Gefangenen, insgesamt eine milde Behandlung erfuhren. Gewiss gab es auch Folterungen, wie sie Hans Schmidt erfuhr, der als Sendbote aus Mähren das Land bereiste. Darüber ist von ihm ein Bericht erhalten ${ }^{63}$ Dergleichen wird aber die Ausnahme gewesen sein. Für wichtiger nahm man den Unterricht durch maßgebende Theologen, die dabei oftmals große Geduld bewiesen. Für die Ortsgemeinden wird man in Rechnung stellen müssen, dass in dem durch die Visitation vermittelten Instanzenweg der hierarchischen Kirchenordung eine Meldung nach oben nur dann rätlich erschien, wenn dies unumgänglich war. Jedenfalls besteht der größte Teil des Bossertschen Quellenwerks vor allem für die Zeit nach dem Tod von Herzog Christoph 1568 und Brenz 1570 aus der Dokumentation von Verhandlungen und Disputationen zwischen Täufern und Theologen.

\section{Weitere Zentren des Täufertums}

Da wir uns hier auf Oberdeutschland beschränkt haben, ist noch auf andere Zentren des Täufertums hinzuweisen. So auf Südtirol, von wo Jakob Huter († 1536) stammt, der ein wichtiges Haupt der täuferischen Bewegung war. Seinen Namen tragen die vielgenannten Hutterischen Brüder in Mähren, die mit ihren Bruderhöfen schließlich dem Druck der Gegenreformation weichen mussten und über Oberungarn und Siebenbürgen nach Russland wanderten, von wo sie 1874 mit 800 Personen nach Nordamerika zogen, wo sie nach wie vor auf ihren Bruderhöfen leben. ${ }^{64}$ Bemerkenswert ist, dass sie ihre Geschichtsbücher bis in die Gegenwart retten konnten. ${ }^{65}$ Es zeigt sich hier, dass dieses Geschichtsbewusstsein eine zentrale identitätsstiftende Funktion hatte und immer noch hat.

60 Ebd., Nr. 961, S. 675.

61 Ebd., Nachtrag Nr. 141 f., S. $1114 \mathrm{ff}$.

62 Ebd., Nr. 1018, S. 760 ff.

63 Ebd., Nr. 947, S. 652-665. Vgl. dazu Hermann Ehmer: Im Turm der Burg Reichenberg. Der Bericht eines Gefangenen aus dem Jahre 1590. In: Unsere Heimat (Beilage der Backnanger Kreiszeitung) $1972 \mathrm{Nr}$. 1.

64 Eine Darstellung des Stands von 1970 bietet: William Albert Allard: The Hutterites, Plain People of the West. In: National Geographic 138 No. 1, July 1970, S. 98-125.

65 Rudolf Wolkan: Geschichtbuch der Hutterischen Brüder. Wien 1923; Andreas J. Friedrich Zieglschmid: Die älteste Chronik der Hutterischen Brüder, Ithaca/NY 1943; Ders.: Das Klein-Geschichtsbuch der Hutterischen Brüder. Philadelphia/Pa. 1947. 
In der Schweiz und im Elsass, besonders in und um Markirch (Ste.-Marie-auxMines) sammelten sich die Anhänger von Jakob Amann, die ebenfalls in die USA auswanderten, wo sie als Amische (Amish) ihr Zentrum in Intercourse im Lancaster County in Pennsylvanien haben, aber auch anderwärts zu finden sind. Sie bilden die weitaus bekannteste religiöse Sondergruppe in den USA, die mit ihren altertümlichen Trachten, ihren Pferdewägelchen und ihrer Präsenz auf den Farmermärkten inzwischen zur amerikanischen Folklore gehören. ${ }^{66}$ Die Amischen sind auch schon Gegenstand von Spielfilmen geworden. Am bekanntesten ist „Witness“ (Paramount 1965, deutsch: Der einzige Zeuge).

Die Amischen haben als Liederbuch den „Ausbund“ ${ }^{67}$ der unter seinen 140 Liedern auch solche von Felix Manz, Michael Sattler und Hans Hut enthält, aber auch eines, das die sieben Brüder im Gefängnis in Schwäbisch Gmünd gemacht haben, ,jeder ein Gesetz" [Strophe]. Auch Lieder, die das Martyrium von Täufern beschreiben, finden sich hier. So eines über Jörg Wagner, der 1527 in München verbrannt wurde. Damit wird das Andenken dieser täuferischen Märtyrer wachgehalten. ${ }^{68}$

Eine weit ausgebreitete Wirksamkeit entwickelte Melchior Hoffmann (ca. 15001543), der zwar aus Schwäbisch Hall stammte, sich aber anderwärts dem Täufertum anschloss. Er wirkte in Livland als Prediger, der sich vom Spiritualisten zum Chiliasten entwickelte. Er trat auch in Dänemark und Norddeutschland auf, wurde dann aber in Straßburg inhaftiert, wo er im Gefängnis starb. ${ }^{69}$

Ein Sonderfall ist die Täuferherrschaft in Münster in Westfalen 1533/34, wo durch Einflüsse aus den Niederlanden ein endzeitliches Königtum errichtet wurde, das aber durch eine Fürstenkoalition beseitigt wurde..$^{70}$ Die Ereignisse von Münster dienten stets als Vorwand, von den Täufern Aufruhr und Empörung zu erwarten.

Nach Menno Simons in den Niederlanden nennen sich die Mennoniten. ${ }^{71}$ Aufgrund der Erfahrung von Münster hatte sich Menno konsequent für die Friedfertigkeit entschlossen. Aber auch die Mennoniten haben sich, bedingt durch Verfolgungen, weiter verbreitetet und Siedlungen in Westpreußen, dann auch in Russland gegründet. Von dort fanden ebenfalls Auswanderungen nach Nordame-

66 Vgl. Lydie Hege, Christoph Wiebe (Hg.): Les Amish. Origine et particularismes 1693-1993. Actes du colloque international de Sainte-Marie-aux-Mines, 19-21 août 1993. Ingersheim 1996.

67 Dem Verfasser lag vor: Ausbund das ist: Etliche schöne Christliche Lieder. Wie sie in dem Gefängnis zu Passau in dem Schloß von den Schweizer-Brüdern und von anderen rechtgläubigen Christen hin und her gedichtet worden. Lancaster County, Pa. ${ }^{13} 1977$.

68 Hermann Ehmer: Die Lieder der Täufer. In: Martin Walser: Das Schauspiel. Szenen aus dem 16.Jahrhundert. Mit Materialien. Hg. von Werner Brändle. Frankfurt 1978, S. 346 f.

69 Klaus Deppermann: Melchior Hoffmann. Soziale Unruhen und apokalyptische Visionen im Zeitalter der Reformation. Göttingen 1979. - Vgl. auch Gerd Wunder: Die Bürger von Hall. Sozialgeschichte einer Reichsstadt 1216-1802 (FWFr 16). Sigmaringen 1980, S. 107 f. Wunder macht ebenda S. 84 eine Verwandtschaft von Melchior Hoffmann mit Margarete Gräter, der ersten Ehefrau von Brenz wahrscheinlich.

70 Kaufmann (wie Anm. 6), S. 327-335.

71 Ebd., S. $334 \mathrm{f}$. 
rika, dann auch nach einigen Staaten Südamerikas statt. Die westpreußischen Mennonitengemeinden wurden durch den Ausgang des Zweiten Weltkriegs zerstört. Die meisten Gemeindeglieder wanderten in die USA und nach Kanada aus, etliche bildeten in Backnang eine bis in die Gegenwart bestehende neue Gemeinde. ${ }^{72}$

Kennzeichnend für den größten Teil der Mennoniten ist die Kriegsdienstverweigerung, die zuzeiten Verfolgungen und Benachteiligungen auslöste. Die Mennoniten haben ebenfalls eine eigene Geschichtsschreibung hervorgebracht, den von Tielemann Janszoon van Braght (1625-1684), dem Ältesten der Mennonitengemeinde von Dordrecht 1660 erstmals herausgegebenen „Märtyrerspiegel“, der die Martyrien der Taufgesinnten vom Anfang des Christentums bis auf seine Gegenwart beschreibt. ${ }^{73}$ Es finden sich dort vor allem Berichte über die niederländischen Täufer, doch wird auch der Märtyrertod von Michael Sattler und Balthasar Hubmaier beschrieben, desgleichen das Gmünder Täufergericht. Erwähnt wird auch Georg Steinmetz, der 1530 in Pforzheim enthauptet wurde, der auch mit einem Lied im „Ausbund“ vertreten ist.

Als neue Gemeinschaft, in der das Täufertum der Reformationszeit wieder auflebte, erscheinen die Baptisten, die aus den englischen Puritanern des 16. Jahrhunderts erwuchsen. Der Begründer des neueren Baptismus in Deutschland ist Johann Gerhard Oncken (1800-1884) von Hamburg, der diesen auch nach Württemberg brachte, wo es alsbald zu baptistischen Gemeindebildungen kam. Diese entstanden hierzulande nahezu gleichzeitig mit den methodistischen Gemeinden. Doch ist dies ein Thema der Konfessionskunde.

72 Horst Klassen: Die Backnanger Mennoniten. Von Galizien, Preußen und Rußland nach Württemberg, Backnang ${ }^{2} 1987$.

73 Dem Verfasser lag vor: Der blutige Schauplatz, oder Märtyrer-Spiegel der Taufgesinnten oder Wehrlosen Christen. Hg. von Thieleman Janszoon van Braght. Aylmer/Ontario und La Grange/Indiana ${ }^{6} 1973$. - Der ursprünglich in niederländischer Sprache verfasste Märtyrerspiegel kam erstmals 1748/49 in Ephrata/Pennsylvanien in deutscher Sprache heraus. Die dort ansässige Gemeinschaft des aus Eberbach am Neckar stammenden Konrad Beisel besorgte die Übersetzung und den Druck. Vgl. dazu Hermann Ehmer: Der ausgewanderte Pietismus. Pietistische Gemeinschaftsprojekte in Nordamerika. In: Rainer Lächele (Hg.): Das Echo Halles. Kulturelle Wirkungen des Pietismus. Tübingen 2001, S. 315-357. 